\title{
Variación de las propiedades térmicas de polvos de carbón en función de la humedad y la distribución del tamaño de grano $^{1}$
}

Jorge Hernando Bautista Ruiz ${ }^{2}$

Recibido:

Junio 4 de 2012

Aceptado:

Noviembre 16 de 2012

${ }^{1}$ Esta investigación fue financiada por el Fondo de Investigaciones Universitarias -FINU - de la Universidad Francisco de Paula Santander

en el marco del desarrollo de proyecto Efecto de la presión de compactación en las propiedades termofísicas de polvos de carbón. Contrato 006 de 2009.

${ }^{2}$ Licenciado en Física y Matemáticas, UPTC. Magister en Metalurgia y Ciencia de los Materiales, UPTC.

Doctor (candidato) en Ingeniería: Ciencia y Tecnología de Materiales, Universidad Nacional de Colombia

Docente - investigador Universidad Francisco de Paula Santander. San José de Cúcuta, Colombia. Correo electrónico:

jorgebautista@ufps.edu.co.

${ }^{3}$ Físico, UPTC. Magister en Metalurgia y Ciencia de los Materiales, UPTC. Doctor en

Ingeniería de los Materiales, Universidad del Valle. Docente investigador Universidad Militar Nueva Granada. Bogotá, Colombia. Correo electrónico:

william.aperador@unimilitar.edu.co.

${ }^{4}$ Físico, UNAL. Magister en Física, Universidad Nacional de Colombia.

Docente - investigador Escuela

Colombiana de Ingeniería "Julio

Garavito". Bogotá, Colombia. Correo electrónico:

aura.morales@escuelaing.edu.co
William Aperador Chaparro $^{3} \quad$ Aura Sofía Mejía ${ }^{4}$
Diciembre 2012

ISSN 0122-820X

PP: 93-101

\section{Resumen}

El propósito de este trabajo fue determinar el efecto de la cantidad de agua en las propiedades termofísicas de muestras de polvo de carbón con diferentes granulometrías. Se reporta el calor específico por unidad de volumen ( $\rho$ ) y la conductividad térmica $(k)$ a temperatura ambiente de polvos de carbón térmico al agregarles agua entre el 5\% y 50\%, porcentaje peso (\%wt). Las muestras fueron obtenidas de la mina Cerro Tasajero ubicada en el área metropolitana de Cúcuta, pulverizadas utilizando molino de rodillos y tamizados en malla pasante números $30(595 \mu \mathrm{m}), 50(297 \mu \mathrm{m})$ y $100(149 \mu \mathrm{m})$. Las propiedades termofísicas se determinaron aplicando el principio físico de flujo lineal transitorio de calor mediante el sistema KD2. El análisis de $k$ y $\rho$ c se realizó ajustando los datos experimentales a una función logística del tipo $y=\frac{\left(A_{1}-A_{2}\right)^{n}}{\left[1-\left(x / x_{0}\right)^{n}\right]}+A_{2}$. Se encontró que las propiedades termofísicas mejoran al aumentar el porcentaje de agua agregado a las muestras y al disminuir el tamaño de grano de los polvos de carbón.

Palabras clave: carbón, distribución de tamaño de grano,función logística, granulometría, KD2, propiedades térmicas.

\section{Abstract}

The aim of this study was to determine the effect of water on the thermophysical properties of specimens of coal powder. We report the specific heat per unit volume $(\rho c)$ and thermal conductivity $(k)$ at room temperature of thermal coal powder when adding water between $5 \%$ and 50\%, weight percent (wt \%). Specimens were obtained from the Cerro Tasajero mine located in Cucuta, pulverized using a roller mill and sieved through-mesh numbers $30(595 \mu \mathrm{m}), 50(297 \mu \mathrm{m})$ and $100(149 \mu \mathrm{m})$. The thermophysical properties were determined using the physical principle of linear transient heat flow through the system KD2. The analysis of $k$ and $\rho_{A}$, , was performed by fitting the experimental data to a logistic function $=\frac{\left(A_{1}-A_{2}\right)}{\left[1-\left(x / x_{0}\right)^{n}\right]}+A_{2}$. It was found that the thermophysical properties improved by 
increasing the percentage of water added to the samples and by decreasing the grain size of coal powder.

Keywords: coal, grain size distribution, KD2, logistic function, thermal properties.

\section{Introducción}

$\mathrm{L}$ a formación del carbón ha sido descrita como "una ineficiencia en el ciclo del carbono", [1], cuando el carbono de las plantas se mantiene en los sedimentos terrestres y no se recicla a la atmósfera [2]. El carbón es, por definición, una roca fácilmente combustible que contiene más del $50 \%$ en peso y $70 \%$ en volumen de material carbonoso [3]. Otra definición describe el carbón como combustible sólido, por lo general estratificada, que se originó a partir de la acumulación, el enterramiento, y la compactación de la vegetación parcialmente descompuesta en las anteriores eras geológicas [4].

El carbón ha sido utilizado por más de 3000 años, en China, en Europa en la edad de bronce, y por indios de las llanuras en los Estados Unidos [1,5]. Durante la edad media, el uso del carbón se hizo más común en hornos y fábricas de cerveza, así como en la calefacción doméstica [6]. En 1850, el carbón se utilizó como combustible para la máquina de vapor y se convirtió en la principal fuente de energía hasta 1950 cuando fue superado por el petróleo para su uso en automóviles y por el gas natural para calefacción [1]. Desde 1984, el carbón ha producido alrededor de una tercera parte de la energía consumida en los Estados Unidos [6]. El 92\% del carbón extraído en los Estados Unidos es utilizado para producir más del 50\% de electricidad [7]. Las proyecciones del Departamento de Energía de EE.UU. indican un incremento en el consumo de carbón a medida que aumenta la demanda de electricidad [8]. El carbón es un combustible fósil con grandes reservas repartidas a nivel global. Actualmente el carbón es la mayor fuente de energía primaria utilizada en plantas termoeléctricas, la industria siderúrgica, así como fuente de combustión en los procesos de quema o cocción de productos cerámicos tradicionales como ladrillos, tejas y baldosas [9-11].

A partir de 2005, el consumo mundial de carbón fue 5883 millones de toneladas (MT) de las cuales China consumió 2339 MT, los Estados Unidos 1086 MT e India 493 MT [8]. El consumo mundial de carbón se proyecta un crecimiento de $2,5 \%$ por año a 9583 MT [7]. A pesar de los problemas ambientales asociados con el uso del carbón de minería (drenaje de ácidos, incendios, las emisiones de gases de efecto invernadero, la contaminación del aire, etc), parece que el carbón seguirá siendo una fuente importante de energía para el futuro [1]. El bajo costo y la disponibilidad del carbón permitirá por muchos años utilizarlo como combustible o fuente de energía especialmente en los países en vía de desarrollo [12].

Mientras que los precios de las materias primas como el petróleo muestran grandes fluctuaciones, los precios del carbón han sido relativamente estables [13]. Además, debido a las reservas de carbón a nivel mundial, probablemente el carbón será una fuente de energía clave para el futuro previsible [13]. De acuerdo con un informe de la Administración de Energía de Estados Unidos, la cantidad de carbón que se consume en todo el mundo se estima que alcanzará 206,3 billones de toneladas en 2035 [14]. En los Estados Unidos, aproximadamente el 90\% del carbón se utiliza para la producción de energía a través de la combustión del carbón [13]. 
Colombia, tiene concentradas sus reservas carboníferas y su explotación en la Costa Atlántica y en el interior del país. De dichas zonas se conocen a nivel nacional siete regiones productoras de carbón, las cuales poseen cerca del $95 \%$ de las reservas totales conocidas. Éstas regiones comprenden los departamentos de La Guajira, Cesar, Córdoba, Santander, Cundinamarca, Boyacá, Antioquia, Valle del Cauca y Norte de Santander [15]. Sobresalen las zonas de Norte de Santander, Cundinamarca y Boyacá por poseer las mayores reservas de carbón metalúrgico y térmico para el consumo nacional y de exportación, con un nivel de producción durante el 2004 de 3,4 MT. La producción total de carbón en el país registró un crecimiento permanente durante el periodo de estudio, representando aumentos notorios del orden de 17 MT entre 1980 1990 y de 32 MT entre 1990 - 2004 [12]. En los últimos años Colombia ha aumentado su participación en las exportaciones de carbón térmico y carbón coquizable, con una buena contribución de la zona de Norte de Santander [16].

El área metropolitana de San José de Cúcuta es rica en suelos arcillosos los cuales proporcionan la materia prima para la elaboración, de índole artesanal, de materiales de construcción como bloque, teja y ladrillo. El proceso de cocción o quema de los productos se hace a través de hornos tipo colmena de llama invertida, cuya fuente principal de combustión es el carbón. En este tipo de hornos el trabajo no es continuo, funciona con tiro natural; es decir el aire de exceso es impulsado de forma natural por diferencias de alturas entre el lecho del horno y la chimenea y no por un ventilador. La alimentación del horno se realiza por bocas laterales y su capacidad depende del diámetro que generalmente está entre 3 y 15 metros, y sus paredes son de aproximadamente un metro de espesor [17].

La fabricación artesanal de materiales para la construcción cuya base es la arcilla, presenta varios inconvenientes relacionados con el tipo de horno, los quemadores utilizados, la eficiencia de los procesos y los gases de combustión que inciden en la calidad del producto y en las condiciones ambientales de trabajo [17].

Debido a lo representativo del sector cerámico en Norte de Santander, se están desarrollando estudios que permitan optimizar los procesos de producción de materiales empleados en la construcción. El objetivo principal es lograr tecnologías más limpias que mejoren tanto la eficiencia, aumentando la cantidad de energía aprovechada, como la tolerancia ambiental en el empleo del carbón. En este trabajo se presentan algunos resultados parciales del efecto que produce la presión de compactación de los polvos de carbón térmico en cuanto a las propiedades termo-físicas de muestras extraídas de las minas de carbón más representativas del área metropolitana de San José de Cúcuta. Los resultados encontrados en el desarrollo de esta investigación serán empleados en la fabricación de cilindros de carbón, previamente beneficiados y compactados a altas presiones. Estos cilindros se emplearán, muy posiblemente, como combustible de los hornos tipo colmena ubicados en San José de Cúcuta y área fronteriza con la República Bolivariana de Venezuela.

\section{2. procedimiento experimental}

\subsection{Preparación de las muestras}

Se tomaron muestras representativas de carbón mineral de la mina Cerro Tasajero ubicada en el área metropolitana de San José de Cúcuta. Las muestras se pulverizaron manualmente mediante molino de rodillos. Los polvos obtenidos se tamizaron en malla pasante números $30(595 \mu \mathrm{m}), 50(297 \mu \mathrm{m})$ y $100(149 \mu \mathrm{m})$. Posteriormente se desarrolla un proceso de secado en un horno de circulación 作 de $312,0 \pm 0,5 \mathrm{~K}\left(\sim 40^{\circ} \mathrm{C}\right)$ por un tiempo de 
24 horas. A la masa inicial de la muestra, se le agregó agua destilada en porcentaje en peso con variaciones cada $5 \%$ hasta alcanzar un $50 \%$. Cada una de las muestras húmedas se compactaron a una presión de 1,7 $\pm 0,2 \mathrm{MPa}$. Para las mediciones de conductividad térmica y calor especifico por unidad de volumen, se empleó el sistema de flujo transitorio de calor KD2 ${ }^{\circledR}$ Thermal Properties Analyzer (Decagon Devices, Inc). Se tomaron cinco lecturas de $k$ y $\rho c$, con una diferencia entre medidas de cinco minutos y luego se promediaron. La densidad ( $\rho$ ) se determinó como la razón masa volumen para la muestras compactadas. Para complementar la caracterización de las muestras de carbón de la mina Cerro Tasajero, el porcentaje de ceniza se halló por norma ASTM D 3174 [18]; la concentración de azufre por la norma ASTM 3177 [19], mientras que la materia volátil se determinó aplicando la norma ASTM 3175 [20].

\subsection{Método experimental}

El sistema KD2-Pro ${ }^{\circledast}$ permite determinar las propiedades térmicas, aplicando como principio físico el flujo lineal transitorio de calor monitoreando el perfil de temperatura en función del tiempo. El sistema realiza mediciones experimentales, que se ajustan a funciones exponenciales integrales mediante un procedimiento no lineal de mínimos cuadrados. Un término de la tendencia lineal corrige los cambios de temperatura de la muestra durante la medida optimizando así la exactitud de las lecturas [21].

La figura 1 muestra el sistema KD2-Pro ${ }^{\circledR}$ y la disposición experimental empleada para efectuar las mediciones de las propiedades térmicas de los polvos de carbón compactados a diferentes porcentajes de agua y tamaños de grano. Se visualiza la ubicación de la sonda o sensor sobre la muestra de carbón y su conexión al analizador KD2-Pro®.

Los valores de $k$ se determinan mediante el monitoreo de la disipación del calor proveniente de una fuente lineal transitoria dada por un voltaje conocido. El gradiente de temperatura para un medio infinito, a una distancia $r$ (dado en metros) próxima a la fuente lineal de calor, está dado por la ecuación 1 [21].

$$
\Delta T=\frac{Q}{4 \pi k t} e^{\left(-\frac{r^{2}}{\Delta D t}\right)}
$$

Figura 1. Montaje experimental del Sistema KD2-Pro.

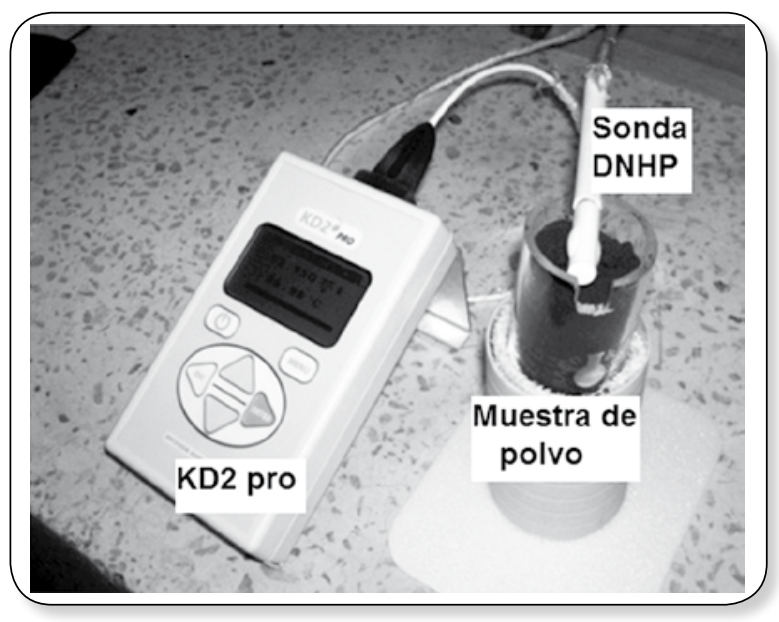

Fuente: presentación propia de los autores

Donde $k$ es la conductividad térmica ( $\mathrm{W} \mathrm{m}^{-1} \mathrm{~K}^{-1}$ ), $\mathrm{D}$ es la difusividad térmica $\left(\mathrm{m}^{2} \mathrm{~s}^{-1}\right), \mathrm{Q}\left(\mathrm{J} \mathrm{m}^{-1}\right)$ corresponde al calor aplicado, y $t$ (s) es tiempo. Considerando a Q como un pulso instantáneo de calor en un intervalo de tiempo $\left(0<\mathrm{t}<\mathrm{t}_{1}\right)$, la ecuación (1) se transforma en la ecuación 2.

$$
\Delta T=-\frac{q}{4 \pi k} E i\left(-\frac{r^{2}}{4 D t}\right) \quad 0<t<t_{1}
$$

Donde $q$ es la tasa de disipación de calor $(\mathrm{W} / \mathrm{m}), t_{1}$ es el tiempo de calentamiento, y $E i$ es una integral exponencial. El gradiente de temperatura después de interrumpir el pulso de calor en el proceso de enfriamiento $\left(t>t_{1}\right)$ está dado por la ecuación 3:

$$
\Delta T=-\frac{q}{4 \pi k}\left[-E i\left(-\frac{r^{2}}{4 D t}\right)+E i\left(-\frac{r^{2}}{4 D\left(t-t_{1}\right)}\right)\right] t>t_{1}
$$

La conductividad térmica de la muestra en estudio, se determina ajustando los datos de temperatura tomados durante el tiempo 
de calentamiento, ecuación (2), y durante el enfriamiento, ecuación (3). Dicho ajuste es no lineal de mínimos cuadrados en los cuales los valores de $\boldsymbol{k}$ se minimizan mediante diferencia entre el modelado y el medido por el sensor de temperatura. Un factor de tendencia lineal se incluye en el procedimiento inverso. La mayoría de los experimentos no ocurren bajo condiciones constantes de temperatura, por esto, se asume que la temperatura puede ser de tendencia lineal y se utiliza un parámetro de ajuste a esta tendencia permitiendo reducir el error sustancialmente.

Otro estudio teórico permite hallar $\boldsymbol{k}$, donde se considera la fuente pulsada de forma cilíndrica de radio $a(\mathrm{~m})$ y longitud $2 b$ (m), midiendo la temperatura en el centro de la muestra. Reporta que el gradiente de temperatura durante el calentamiento $\left(0<t<t_{1}\right)$ es dado por la ecuación (4), $y$ durante el enfriamiento $\left(t>t_{1}\right)$ por la expresión (5)

$$
\begin{aligned}
& \Delta T=\frac{q}{4 \pi k} \int_{r^{2} / 4 D t}^{\infty} u^{-1} \exp (-u) \exp \left[-(a / r)^{2} u\right] \\
& I_{0}(2 a u / r) \operatorname{erf}\left(\frac{b}{r} \sqrt{u}\right) d u \\
& \Delta T=\frac{q}{4 \pi k} \int_{r^{2} / 4 D t}^{r^{2} / 4 D\left(t-t_{1}\right)} u^{-1} \exp (-u) \exp \left[-(a / r)^{2} u\right] . \\
& I_{0}(2 a u / r) \operatorname{erf}\left(\frac{b}{r} \sqrt{u}\right) d u
\end{aligned}
$$

Donde, $I_{0}(x)$ representa una función de Bessel modificada de orden cero, erf $(x)$ es la función error, y $u$ es una variable de integración.

$\mathrm{Si} a / r$ se aproxima a cero, $\mathrm{y} b / r$ a infinito, se reducen las ecuaciones (4) y (5) a las expresiones (2) y (3).

Para el tipo de sensor y microcontrolador que maneja el sistema KD2, se ha encontrado que las ecuaciones (2) y (3) ajustan los datos del gradiente de temperatura tan bien como las ecuaciones (5) y (6), pero dan valores un poco diferentes para el ajuste en los parámetros, variación que debe incluirse en la calibración del equipo, así las ecuaciones más simples (2) y (3) son confiables para determinar el valor de $k$.

\section{Resultados y discusión}

Las figuras 2 y 3 muestran las gráficas de la conductividad térmica y el calor específico por unidad de volumen, respectivamente. En cada caso se visualiza el comportamiento en función de los tres tamaños de grano y la cantidad de agua agregada a las muestras. La temperatura promedio a la cual se desarrollaron los análisis fue de $229,0 \pm 0,3 \mathrm{~K}$.

Figura 2. Conductividad térmica en función del porcentaje de agua agregado a la muestra para las tres granulometrías.

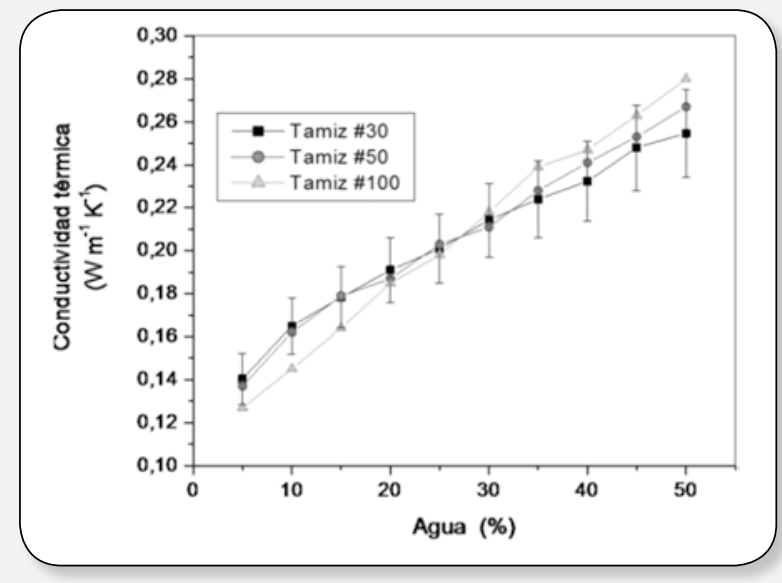

Fuente: presentación propia de los autores

El comportamiento de $k$ y $\rho c$ en función de la concentración de agua (\% wt), figuras 2 y 3 respectivamente, de éstas se aprecia que tanto $\boldsymbol{k}$ como $\rho c$ aumentan al incrementarse la concentración de agua. Este mismo comportamiento se presenta para las tres granulometrías estudiadas. ISSN 0122-820X 
Figura 3. Calor específico por unidad de volumen en función del porcentaje de agua agregado a la muestra para las tres granulometrías.

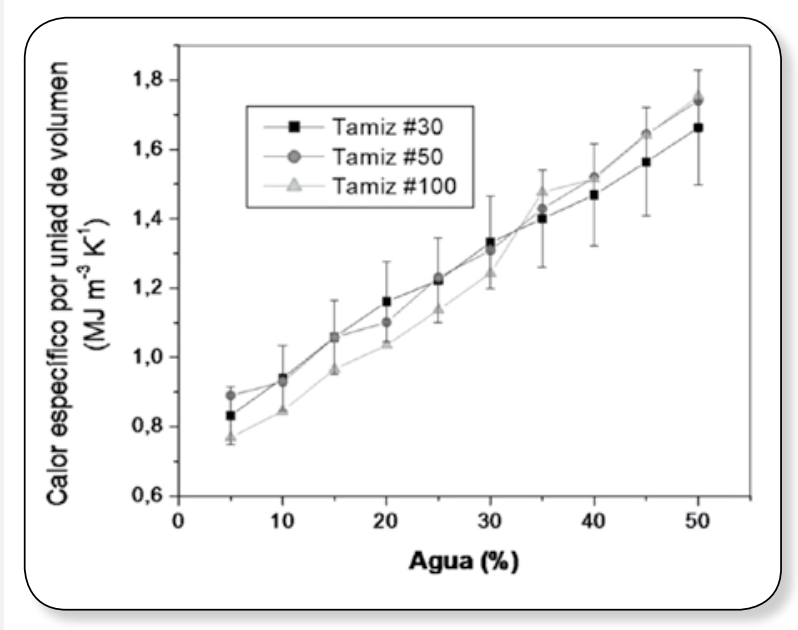

Fuente: presentación propia de los autores

Para las muestras de carbón con tamaño de grano inferior a $297 \mu \mathrm{m}$ (pasante tamiz \# 50) el valor de $\boldsymbol{k}$ a una concentración de agua del 50\% Wt es el 94,89\% mayor que cuando tiene $5 \%$ wt de agua agregada. pc se incrementa en un $95,62 \%$ entre la condición inicial (5\%wt) $y$ final (50\%wt).

Y finalmente, para las muestras de carbón con tamaño de grano menor a $149 \mu \mathrm{m}$ (pasante tamiz \#100) el valor de $\boldsymbol{k}$ al 50\% wt de agua es $139,31 \%$ mayor que para $5 \%$ wt de agua agregada. $\rho c$ se incrementa en un $128,08 \%$ entre la condición inicial (5\%wt) y final $(50 \% w t)$.

De los análisis anteriores es posible concluir, parcialmente, que las mejores propiedades termofísicas las ofrece las muestras de carbón con tamaño de grano de $149 \mu \mathrm{m}$ equivalente a partículas pasantes malla \#100.

Los resultados anteriormente relacionados son coherentes pues se encontró que las densidades de la muestras aumentan al incrementarse la concentración de agua, tal como se aprecia en la figura 4.

En las figuras 2 y 3, se observan las gráficas de los datos experimentales para los parámetros termofísicos $(\boldsymbol{k}$ y $\rho c)$ en función de la concentración (\%wt) de agua agregada a la muestra. Estas gráficas se ajustaron a una función logística de crecimiento con límite de saturación dada por la expresión (5) [22].

$$
y=\frac{\left(A_{1}-A_{2}\right)}{\left[1-\left(x / x_{o}\right)^{n}\right]}+A_{2}
$$

En la ecuación 5, $A_{1}$ corresponde al valor de la propiedad térmica $(y)$ de la muestra cuando no se agrega agua $(x=0 \% w t) ; A_{2}$ corresponderá al valor de la propiedad térmica cuando la concentración de agua es muy grande $(x>>)$, es decir el valor máximo de saturación; $x_{0}$ corresponde al punto donde se produce el cambio de curvatura y $n$ es la razón de crecimiento. En las tablas 1 y 2 se relacionan los valores de cada uno de los parámetros de la función logística para $k$ y rc. También se registran los parámetros estadísticos de correlación.

Figura 4. Densidad en función de la concentración de agua (\%wt) agregada a la muestra.

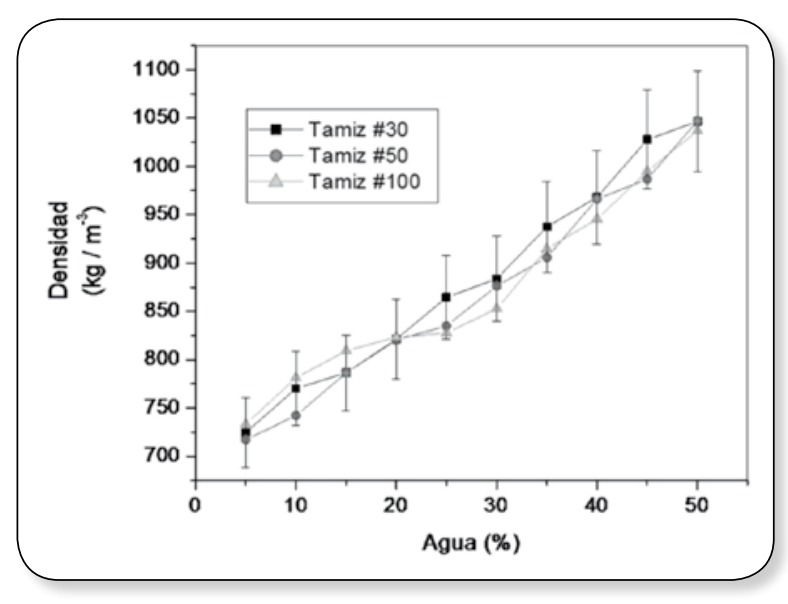

Fuente: presentación propia de los autores

Tabla 1. Valores de los parámetros de la función logística para $k$ de los polvos de carbón en los tres tamaños de grano.

\begin{tabular}{|c|c|c|c|c|}
\hline $\begin{array}{c}\# \\
\text { Tamiz }\end{array}$ & $\mathrm{A}_{1}$ & $\mathrm{~A}_{2}$ & $\mathrm{X}_{0}$ & $\mathrm{n}$ \\
\hline 30 & $0,137 \pm 0,001$ & $0,494 \pm 0,003$ & $94,376 \pm 0,005$ & $1,121 \pm 0,001$ \\
\hline 50 & $0,123 \pm 0,003$ & $0,717 \pm 0,008$ & $89,934 \pm 0,003$ & $1,004 \pm 0,007$ \\
\hline 100 & $0,109 \pm 0,002$ & $0,609 \pm 0,001$ & $87,074 \pm 0,002$ & $1,207 \pm 0,002$ \\
\hline
\end{tabular}

Fuente: presentación propia de los autores 
Variación de las propiedades térmicas de polvos de carbón en función de la

Tabla 2. Valores de los parámetros de la función logística para $\rho c$ de los polvos de carbón en los tres tamaños de grano.

\begin{tabular}{|c|c|c|c|c|}
\hline $\begin{array}{c}\# \\
\text { Tamiz }\end{array}$ & $\mathrm{A}_{1}$ & $\mathrm{~A}_{2}$ & $\mathrm{X}_{0}$ & $\mathrm{n}$ \\
\hline 30 & $0,708 \pm 0,005$ & $8,068 \pm 0,006$ & $93,993 \pm 0,003$ & $0,922 \pm 0,005$ \\
\hline 50 & $0,834 \pm 0,003$ & $6,133 \pm 0,002$ & $87,247 \pm 0,006$ & $1,433 \pm 0,007$ \\
\hline 100 & $0,734 \pm 0,001$ & $4,088 \pm 0,001$ & $83,361 \pm 0,002$ & $1,601 \pm 0,009$ \\
\hline
\end{tabular}

Fuente: presentación propia de los autores

De lo anterior se encontró: que para polvos de carbón con tamaño de grano inferior a 595 $\mu \mathrm{m}$ (pasante tamiz \#30), el valor de $k$ y $\rho c$ para cuando no se agrega agua a la muestra es de $0,137 \mathrm{Wm}^{-1} \mathrm{~K}^{-1}$ y $0,708 \mathrm{MJ} \mathrm{m}^{-3} \mathrm{k}^{-1}$, de igual forma los valores para estos parámetros termofísicos cuando hay saturación de agua reportó un valor de $0,494 \mathrm{Wm}^{-1} \mathrm{~K}^{-1}$ para $k \mathrm{y}$ $8,068 \mathrm{MJ} \mathrm{m}^{-3} \mathrm{k}^{-1}$ para rc.

Para polvos con tamaño de grano inferior a $297 \mu \mathrm{m}$ (pasante tamiz \#50), el valor de $k$ y $\rho$ c para cuando no se agrega agua a la muestra es de $0,123 \mathrm{Wm}^{-1} \mathrm{~K}^{-1}$ y $0,834 \mathrm{MJ} \mathrm{m}^{-3}$ $\mathrm{k}^{-1}$, cuando hay saturación de agua reportó un valor de $0,717 \mathrm{Wm}^{-1} \mathrm{~K}^{-1}$ para $k$ y $6,133 \mathrm{MJ}$ $\mathrm{m}^{-3} \mathrm{k}^{-1}$ para rc.

Y finalmente, para polvos con tamaño de grano inferior a $149 \mu \mathrm{m}$ (pasante tamiz \#100), el valor de $k$ y $\rho c$ para cuando no se agrega agua a la muestra es de $0,109 \mathrm{Wm}^{-1} \mathrm{~K}^{-1}$ y 0,734 $\mathrm{MJ} \mathrm{m}^{-3} \mathrm{k}^{-1}$, cuando hay saturación de agua reportó un valor de $0,609 \mathrm{Wm}^{-1} \mathrm{~K}^{-1}$ para k y 4,088 $\mathrm{MJ} \mathrm{m}^{-3} \mathrm{k}^{-1}$ para $\rho c$.

Para complementar el estudio, los polvos de carbón de la Mina Cerro Tasajero se caracterizaron químicamente. De tal forma que el porcentaje de ceniza presente en la muestra, se determinó mediante la norma ASTM D 3174; la concentración de azufre se establece con la norma ASTM 3177, reportando un valor de $0,45 \%$, mientras que la materia volátil se precisa aplicando la norma ASTM 3175. Esta información se encuentra registrada en la tabla 3.
Tabla 3. Valores de los análisis químicos de las muestras de carbón térmico.

\begin{tabular}{|c|c|}
\hline Análisis & \% \\
\hline Ceniza & $1,75 \pm 0,03$ \\
\hline $\begin{array}{c}\text { Material } \\
\text { volátil }\end{array}$ & $34,17 \pm 0,05$ \\
\hline Azufre & $0,45 \pm 0,02$ \\
\hline
\end{tabular}

Fuente: presentación propia de los autores

Los resultados presentados sugieren, que en los hornos la tasa de combustión se ve favorecida debido al aumento de la temperatura por incidencia directa de la capacidad calorífica y la conductividad térmica, como lo sugiere Maloney [23] y colaboradores.

\section{Conclusiones}

De los tres tamaños de grano estudiados, se encontró que los mejores resultados en las propiedades termofísicas se obtuvieron para polvos de carbón con tamaño de grano de $149 \mu \mathrm{m}$ (tamiz \#100) en donde el valor de $\boldsymbol{k}$ a una concentración de agua del 50\% wt es el 139,31\% mayor que al 5\% wt. pc se incrementa en un 128,08\% entre la condición inicial $(5 \% w t)$ y final $(50 \% w t)$.

El porcentaje de ceniza, presente en la muestra, se determinó mediante la norma ASTM D 3174 encontrándose un valor de 1,75\%; una concentración de azufre 0,45\% según la norma ASTM 3177 y 34,17\% de materia volátil aplicando la norma ASTM 3175. ISSN 0122-820X 


\section{Referencias \\ bibliográficas}

[1]. KIM, A. Coal Formation and the Origin of Coal Fires. En: Coal and Peat Fires: A Global Perspective.ed. Elsevier: New York, 2011, pp 1-28.

[2]. BARGHOORN, E. Degradation of plant materials and its relation to the origin of coal. 2nd Conf. on the Origin and Constitution of Coal. Nova Scotia, 1952, pp. 181-203.

[3]. SCHOPF, J. Definitions of peat and coal and of graphite that terminates the coal series. J. Geol. 1966, vol. 74, pp. 584592.

[4] HENDRICKS, T. The origin of coal.En: Chemistry of Coal Utilization.ed. New York: John Wiley and Sons Inc.,1945, pp. 1-24.

[5] CASSIDY, S. History of coal mining, Elements of Practical Coal Mining.Coal. 1973, vol. 2, pp. 1-10.

[6]. US Energy Information Administration. International Energy Outlook - Coal, [web en línea]. <http://www.eia.doe. gov/oiaf/ieo/coal.htm $>$. [Consulta: 3-1-2011]

[7]. US Energy Information Administration USA. Annualenergyoutlook [documento en línea]. 2006. [Washington, DC]: 10/12/2002. <http://www.eia. doe.gov/oiaf/ieo/pdf/ieoreftab_6.pdf> [consulta: 10-8-2011]

[8]. US EnergyInformationAdministration USA [web en línea]. <http://www. eia.doe.gov/emeu/international/ coalconsumption.html>. [Consulta: 15-11-2008]
[9] SEHLKE, G. U. S. Department of Energy's Role in the Energy-Water Nexus. Journal of Contemporary Water Research and Education.2009, vol. 143,núm. 2, pp. 56-62.

[10]. HIROAKI, Y. Coal Firing Power Generation Technology. Journal of the Japan Institute of Energy. 2003, vol. 82, pp. 822-829.

[11]. VIEIRA, C. Incorporation of solids wastes in red ceramics - an update review. RevistaMateria. 2009, vol. 14, pp. 881-905.

[12] UNSWORTH, J. Coal Quality and Combustion Performance. An international perspective.Coal Science and Technology.1991, vol. 19, núm. 2, pp. 638-642

[13]. LEE, H.; KLIMA, M.; SAYLOR, P. Evaluation of a laboratory rod mill when grinding bituminous coal. Fuel. 2012, vol. 92, pp. 116-121.

[14]. US Energy Information Administration USA, Energy in the United States: 1635-2000 [documento en línea]. 2001. [Washington, DC]: 12/06/2002 $<$ http://www.eia.doe.gov/emeu/aer/ eh $>$ [consulta: 10-8-2011]

[15]. CIMAC, C. Selección de carbones para la aplicación del combustible CCTA en hornos de cerámica dentro de los esquemas de producción limpia y uso racional de energía- departamentos de Boyacá, Santander y Norte de Santander. Centro de Investigación de Materiales Cerámicos, Univ. Francisco de Paula Santander, 2004. 85 p

[16]. VALBUENA, O. Efecto de la distribución de tamaño de partícula en la conductividad térmica de polvos de carbón usados en la elaboración del combustible tipo CCTA. San José de Cúcuta: Universidad 
Francisco de Paula Santander, 2007. $120 \mathrm{p}$

[17]. TRIVIÑO, M.; MOLINA, C. Diagnóstico técnico de carbones térmicos para la obtención del combustible CCTA en Boyacá. Revista Energética. 2007, vol. 37, pp. 13-22.

[18].AMERICAN Section of the International Association for Testing Materials, ASTM (US). ASTM Standard D3174, 2011. Method for Ash in the Analysis Sample of Coal and Coke from Coal.ASTM, $2011.16 \mathrm{p}$.

[19]. AMERICAN Section of the International Association for Testing Materials,ASTM (US). ASTM Standard D3177 - 02, 2007. Test Methods for Total Sulfur in the Analysis Sample of Coal and Coke. ASTM, 2007. 12p.

[20]. AMERICAN Section of the International Association for Testing Materials,ASTM (US). ASTM Standard D3175, 2011. Test Method for Volatile Matter in the Analysis Sample of Coal and Coke. ASTM, 2011. 15 p.

[21]. DecagonDevices [web en línea]. $<$ http://www.ictinternational.com.au/ kd2.htm > . [Consulta: 5-1-2012]

[22]. DRAGAN, A. The existence of optimal parameters of the generalized logistic function.Applied Mathematics and Computation. 1996, vol. 77, pp. 281 294.

[23]. DANIEL J. MALONEY, D.A.; SAMPATH, R. AND ZONDLO, J. Heat capacity and thermal conductivity considerations for coal particles during the early stages of rapid heating. Combustion and Flame. 1999, vol. 116(1-2), pp. 94-104. 